\title{
Correlación clínico patológica del cáncer cervical y precursores en una población de Lima periférica
}

\author{
Victoria Valer ${ }^{1,2}$, Delia Jara ${ }^{2}$, Gloria Asmat ${ }^{2}$, Diana Tello ${ }^{2}$
}

Resumen

Palabras clave

\begin{abstract}
Objetivo: Determinar la prevalencia y los factores de riesgo del cáncer cervical y sus precursores en un grupo poblacional, con especial énfasis en el manejo de la enfermedad cervical preinvasiva. Diseño: Estudio prospectivo y descriptivo no aleatorio. Materiales y Métodos: Estudio realizado durante el año 2003, en mujeres del Centro de Salud de Piedra Liza (San Juan de Lurigancho) en 120 pacientes con diagnóstico citológico de ASC, AGC, L-SIL, H-SIL y carcinoma; se realizó biopsia de las lesiones cervicales en 49 casos, para confirmar el diagnóstico citológico y correlacionarlo con el cuadro clínico y la colposcopia. Resultados: De los 120 casos de estudio citológico, 14 (11,5\%) fueron ASC; 67 (56\%) L-SIL, 34 (29\%) H-SIL, 3 (2,5\%) carcinoma escamoso y 2 (2\%) adenocarcinoma. De 49 biopsias, 12 casos fueron L-SIL, 32 H-SIL, 3 carcinoma escamoso invasor y 2 adenocarcinoma cervical, uno de ellos in situ. Conclusiones: Los resultados citológicos muestran que las lesiones escamosas intraepiteliales y el carcinoma cervical tuvieron alta prevalencia en este grupo poblacional.
\end{abstract}

Neoplasmas de cuello uterino; biopsia; carcinoma de células escamosas; factores de riesgo.

\section{Cervical cancer and precursors clinical and pathological correlations in a Lima's periphery population}

\section{Abstract}

Objective: To determine both the prevalence and risk factors of cervical cancer and squamous intraepithelial lesions, with special attention on the preinvasive cervical disease treatment. Design: Prospective, descriptive, non randomized study. Materials and Methods: Study of 120 non randomly selected cases of cervical cytology during 2003, in women attended at Piedra Liza Health Center (San Juan de Lurigancho). In 49 cases, biopsy was performed to confirm both clinical and colposcopic findings. Results: From the 120 cytology cases, 14 (11,5\%) were ASC, 67 (56\%) L-SIL, 34 (29\%) H-SIL, 3 (2,5\%) squamous carcinoma, 2 (2\%) adenocarcinoma. In the 49 biopsies, 12 cases were L-SIL, $32 \mathrm{H}$-SIL, 3 invasive squamous carcinoma, and 2 cervical adenocarcinoma, one in situ. Conclusions: Cytology results suggest that squamous intraepithelial lesions and cervical carcinoma have higher prevalence in this study group.

\footnotetext{
1 Departamento de Cirugía Humana. Facultad de Medicina, UNMSM. Lima, Perú.

2 Instituto de Patología. Facultad de Medicina, UNMSM. Lima, Perú.
}

Keywords: Cervix neoplasms; biopsy; carcinoma, squamous cell; risk factors.

\section{INTRODUCCIÓN}

El cáncer del cérvix uterino, en nuestro país, constituye un problema social y de salud, especialmente en mujeres que provienen de estratos socioeconómicos bajos y a pesar de las políticas y programas orientados a disminuir su morbimortalidad.

Lima Metropolitana en el Perú ocupa el segundo lugar en incidencia de cáncer cervical después del cáncer de mama. A nivel de provincias, continúa ocupando el primer lugar $\left({ }^{1-6}\right)$ y su tasa de mortalidad el segundo lugar de todos los cánceres $(9,3 / 100000$ mujeres $)$. Se observa que el promedio de edad del grupo poblacional con cáncer cervical es menor a la referida por autores internacionales.

La incidencia y la prevalencia están relacionadas con múltiples factores de riesgo ya 
conocidos, dentro de los cuales los agentes infecciosos juegan un papel preponderante en su génesis, especialmente el virus papiloma humano (VPH) de alto riesgo -16, 18, 31 y 33-, asociados a otros cofactores infecciosos (como virus herpes simple II, virus citomegálico, Chlamydia trachomatis, etc.), cigarrillo, anticonceptivos, relaciones sexuales a temprana edad, promiscuidad sexual de la pareja, estatus socioeconómico bajo y otros $\left({ }^{7-12}\right)$.

La infección por VPH se adquiere a través de las relaciones sexuales y el blanco de su acción en el cérvix uterino es la zona de transición, donde el ADN viral se va a integrar al genoma humano y las oncoproteínas E6 y E7 producidas por los VPH de alto riesgo van a inactivar la función supresora de los genes p53 y $\mathrm{Rb}$, ocasionando una proliferación celular descontrolada durante su pasaje por el ciclo celular y carcinogénesis.

El cáncer cervical tiene una historia natural de progresión lenta, pasando por lesiones precursoras llamadas lesiones cervicales escamosas intraepiteliales (SIL, siglas en inglés), que se caracterizan por estar confinadas a la mucosa cervical y respetan la membrana basal. El SIL se clasifica en bajo grado (displasia leve e infección por virus papiloma humano) y alto grado (displasia moderada, displasia severa y carcinoma in situ). Estas lesiones pueden ser detectadas tempranamente mediante el método de Papanicolaou y tratadas oportunamente; en caso contrario, la mayoría progresará a carcinoma invasor, el cual tiene un pronóstico sombrío.

Los cánceres cervicales se originan en las células escamosas en 80 a $90 \%$ y 10 a $15 \%$ de los adenocarcinomas se desarrollan en células cilíndricas. El Sistema Bethesda recomienda que, las pacientes con citología anormal de células escamosas atípicas en quienes no se puede excluir lesión intraepitelial de alto grado (ASC-H), deben ser evaluadas mediante estudio histológico de biopsias obtenidas por evaluación colposcópica. El objetivo de nuestro estudio es determinar la prevalencia y los factores de riesgo del cáncer cervical y precursores en este grupo poblacional, con un especial énfasis en el diagnóstico y manejo de la enfermedad preinvasiva del cáncer cervical.

\section{MATERIALES Y MÉTODOS}

Se efectuó un estudio prospectivo, transversal y descriptivo, durante el año 2003, en 2400 mujeres del Centro de Salud de Piedra Liza (San Juan de Lurigancho). El primer estudio de las láminas citológicas fue realizado por una citotecnóloga, quien nos remitió 349 casos determinadas como citologías atípicas. Éstas fueron reevaluadas por un patólogo del Instituto de Patología de la Universidad Nacional Mayor de San Marcos, quien consideró sólo 120 casos como citología atípica sospechosa de malignidad, motivo de nuestro estudio. Se elaboró formatos para incluir los datos de anamnesis y examen clínico de la paciente. Los 120 casos fueron derivados al consultorio de Ginecología de Proyección Social del Instituto de Patología de la UNMSM, para su evaluación, seguimiento y tratamiento. Se incluyó como unidades de análisis las 120 secreciones cervicovaginales y 49 biopsias cervicales del cuello uterino, haciéndose un estudio de correlación citohistológico.

Se tuvo en cuenta el antecedente de toma de muestra de secreción cervicovaginal no mayor de 3 meses, en una lámina previamente rotulada, obtenida con espátula de Ayre; además de la muestra pancervical (ectocérvix, endocérvix y vagina), se obtuvo con citocepillo una muestra endocervical de mucosa profunda, las cuales fueron extendidas homogéneamente, fijadas con alcohol corriente al $95 \%$ y coloreadas según el método de Papanicolaou. Cuando hubo resultados sospechosos de malignidad, se hizo una biopsia dirigida del cérvix, bajo control colposcópico; se fijó en formol al 10\%, a seguir se hizo cortes histológicos de $4 \mathrm{~mm}$ y se tiñó con hematoxilina eosina. Los casos de citología atípica benigna y lesiones intraepiteliales de bajo 
grado recibieron tratamiento médico, previamente a un nuevo control citológico.

Las pacientes que tuvieron diagnóstico histológico de lesión intraepitelial de alto grado y carcinoma cervical fueron derivadas al Instituto Nacional de Enfermedades Neoplásicas. La nomenclatura citológica utilizada fue la de Bethesda Modificada (2001) ${ }^{(13,14}$ ) (Tabla 1), presentada en Vancouver; y, para la clasificación histológica, se usó la de Richart modificada $\left({ }^{14}\right)$ y aceptada por Bethesda.

Tabla 1. Nomenclatura citológica de acuerdo al sistema BETHESDA 2001.

Anormalidades de las células epiteliales escamosas:

A. Células escamosas atípicas (ASC)

1. Células escamosas atípicas de significado no determinado (ASC-US)

- ASC-US: (Atypical squamous cells of undetermined significance, células escamosas atípicas de significado indeterminado o incierto.

- No puede excluirse HSIL (ASC-H)

2. Lesión intraepitelial escamosa de bajo grado (L-SIL)

- Virus papiloma humano (VPH)

- Displasia leve (CIN 1).

3. Lesión intraepitelial escamosa de alto grado (H-SIL):

- Displasia moderada, displasia severa y carcinoma in situ (CIN 2 y 3)

4. Carcinoma escamosa

B. Células glandulares atípicas (AGC)

- Endocervicales (NOS o especificar en comentarios)

- Endometriales (NOS o especificar en comentarios)

- Glandulares (NOS o especificar en comentarios).

Nomenclatura histológica para precursores de carcinoma:

- Lesión escamosa intraepitelial de Bajo Grado (L-SIL):

Neoplasia intraepitelial Grado I (CIN I)

- Lesión escamosa intraepitelial de Alto Grado (H-SIL):

Neoplasia intraepitelial Grados II y III
La interpretación estadística de los resultados aplicó medidas de tendencia central, frecuencias absolutas y porcentajes, que fueron representadas mediante tablas.

\section{RESULTADOS}

El inicio de relaciones sexuales fue antes de los 15 años en $26(21,7 \%), 15$ a 18 años en 49 $(40,8 \%) ; 19$ a 22 en $24(20 \%), 23$ a 25 años en $21(17,5 \%)$. El número de compañeros sexuales fue uno en 20 casos $(16,7 \%)$, dos en $28(23,3 \%)$, tres en $48(40 \%)$, cuatro en $20(16,7 \%)$, cinco en $4(3,3 \%)$. Con relación al grado de instrucción, tuvieron educación superior $1(0,8 \%)$, secundaria completa e incompleta $29(24,2 \%)$, primaria completa e incompleta 90 (75\%). El nivel socio económico fue alto en ningún caso, medio en 8 $(6,7 \%)$, bajo en $112(93,3 \%)$. El estado de nutrición fue bueno en $12(10 \%)$, regular en 40 $(33,3 \%)$, malo en $68(56,7 \%)$. Empleaban anticoncepción, DIU $50(41,7 \%)$, anticonceptivos orales $40(33,3 \%)$, inyectables $11(9,2 \%)$ y preservativos y otros $19(15,8 \%)$.

En los 120 casos, los diagnósticos citológicos fueron (Tabla 2): células escamosas atípicas (ASC) 14; en 5 de ellas, células escamosas atípicas de significado no determinado sugerentes de infección por VPH y en 9 no se pudo excluir H-SIL. Lesiones escamosas intraepiteliales de bajo grado fueron 67,8 con infección por virus papiloma humano (VPH); en 59, neoplasia intraepitelial escamosa grado I (CIN I), 17 asociadas a infección por VPH. Las lesiones intraepiteliales escamosas de alto grado fueron 34; 14 correspondieron a CIN II, 2 de ellas asociadas a infección por VPH y 20 correspondieron a CIN III (displasia severa y carcinoma in situ), 5 de ellas asociadas a infección por VPH. Tres casos correspondieron a carcinoma escamoso invasor, en un caso asociado a VPH. De los dos casos de adenocarcinoma cervical, uno era in situ y el otro invasor.

De las 49 biopsias (Tabla 3), 12 correspondieron a lesiones intraepiteliales 
Tabla 2. Diagnóstico citológico de anormalidades cervicales.

\begin{tabular}{lrr}
\hline \multicolumn{1}{c}{$\begin{array}{c}\text { Anormalidades de las } \\
\text { células epiteliales escamosas }\end{array}$} & $\mathrm{n}$ & $\%$ \\
\hline $\begin{array}{l}\text { 1. Células escamosas atípicas } \\
\text { A) De significado no determinado }\end{array}$ & 14 & 11,5 \\
$\quad$ (ASC-US) & 5 & 4,0 \\
B) No puede excluirse HSIL (ASC-H) & 9 & 7,5 \\
2. Lesiones intraepiteliales escamosas & & \\
de bajo grado & 67 & 56,0 \\
A) Virus papiloma humano & 8 & 7,0 \\
B) NIC I (Displasia leve) & 59 & 49,0 \\
3. Lesiones intraepiteliales escamosas & & \\
de alto grado & 34 & 29,0 \\
A) NIC II (Displasia moderada) & 14 & 12,0 \\
B) NIC III (Displasia severa y & & \\
$\quad$ carcinoma in situ) & 20 & 17,0 \\
4. Carcinoma escamoso invasor & 3 & 2,5 \\
5. Anormalidades de las células & & \\
epiteliales glandulares & 2 & 2,0 \\
a. Adenocarcinoma in situ & 1 & 1,0 \\
b. Adenocarcinoma invasivo & 1 & 1,0 \\
Total & 120 & 100,0 \\
\hline
\end{tabular}

escamosas de bajo grado, 32 a lesiones intraepiteliales escamosas de alto grado. Tres casos de carcinoma escamoso cervical invasor y 2 casos de adenocarcinoma cervical -uno de ellos in situ- correlacionaron con el estudio citológico.

\section{DISCUSIÓN}

Las estadísticas de cáncer cervical en nuestro país son alarmantes, a pesar de la existencia de programas de detección de larga data. El cáncer de cérvix continúa siendo un problema de salud pública, a pesar de existir un método de detección citológica universal, como es el estudio de Papanicolaou, que detecta el cáncer cervical en sus etapas de lesiones intraepiteliales cervicales, lo cual nos permitiría un seguimiento y un tratamiento oportuno.

Reportes, como el Registro de cáncer de Lima Metropolitana $\left(^{5}\right)$, señalan que el cáncer cervical ocupa el segundo lugar después del cáncer de mama. Albújar $\left({ }^{15}\right)$ refiere que en Trujillo es la primera causa de mortalidad de cáncer en mujeres. Nuestro estudio comprende una población de la zona de San Juan de Lurigancho, área conformada por un estrato socioeconómico bajo, donde observamos que la prevalencia del cáncer cervical y precursores es alta y los resultados de los factores de riesgo para carcinogénesis cervical coinciden con lo observado por diferentes investigadores: estatus socioeconómico bajo, relaciones sexuales a temprana edad, promiscuidad sexual de la pareja, anticonceptivos, entre otros, por lo que su prevención en nuestro país debe enfocarse a garantizar servicios costo efectivos de los programas de detección de cáncer. Éstos deben de estar disponibles para estimular la participación masiva de las mujeres de alto riesgo que se encuentran en las áreas de Lima periférica y zonas rurales de Lima y provincias. Estamos de acuerdo con lo referido por Koss y Okagaki $\left({ }^{16,17}\right)$, de que este tipo de cáncer afecta en forma totalmente desproporcionada a las mujeres más humildes, quienes tienen menos posibilidades de acceso a tratamientos efectivos y donde una de las fallas principales de muchos centros detectores de cáncer cervical es la baja confiabilidad del diagnóstico de Papanicolaou, debido a que no reúnen condiciones de calidad. Una de ellas es la insuficiente e inadecuada capacitación del personal, asociada a la falta de recursos humanos, pobreza de información clínica, supervisión $\left({ }^{18}\right)$ y seguimiento de los

Tabla 3. Diagnóstico histopatológico.

\begin{tabular}{lrr}
\hline \multicolumn{1}{c}{ Diagnóstico histológico } & Casos & \% \\
\hline $\begin{array}{l}\text { Lesiones intraepiteliales de células } \\
\text { escamosas de bajo grado (L-SIL) }\end{array}$ & 12 & 24,5 \\
$\begin{array}{l}\text { Lesiones intraepiteliales de células } \\
\text { escamosas de alto grado (H-SIL) }\end{array}$ & 32 & 65,3 \\
Carcinoma escamoso invasor & 3 & 6,1 \\
Adenocarcinoma (in situ) & 1 & 2,0 \\
Adenocarcinoma infiltrante & 1 & 2,0 \\
Total & 49 & 100,0 \\
\hline
\end{tabular}


casos en los centros de detección, así como el uso de diferentes criterios de diagnóstico y de clasificación.

La detección citológica del VPH en nuestro estudio fue en 33 casos, con asociación o no a SIL y carcinoma. Santos $\left({ }^{19}\right)$, en el Perú, señala que el VPH es uno de los agentes infecciosos más frecuentemente relacionados con la etiología del cáncer, junto a diferentes cofactores. El autor realizó un estudio del ADN del VPH en cánceres cervicales infiltrantes, detectando $95,3 \%$ de mujeres con carcinoma a células escamosas y $92 \%$ de mujeres con adenocarcinoma y carcinoma adenoescamoso. Estamos de acuerdo que la detección de VPH debe ser realizado por tecnología de biología molecular, porque no sólo se detecta el ADN del virus, sino también los subtipos de riesgo para desarrollar cáncer, estudio que no se pudo realizar en el Instituto de Patología por no contar con los recursos necesarios para su elaboración. Los exámenes de VPH deben ser realizados en los casos de ASC (células escamosas atípicas de significado no determinado) y en los SIL de bajo grado. Si el resultado molecular de VPH indica que es un tipo de alto riesgo, se debe recomendar biopsia dirigida bajo control colposcópico (Figura 1).

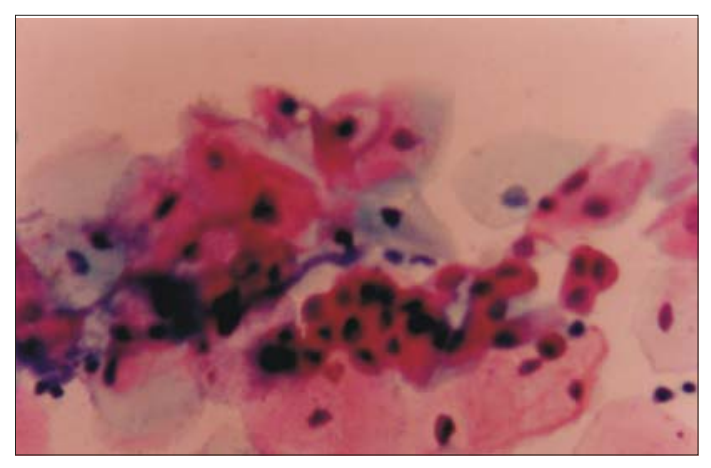

Figura 1. Frotís atrófico con células epiteliales pavimentosas parabasales disqueratósicas con hipercromasia nuclear. Fondo con infiltrado inflamatorio agudo. (Antecedente de uso de inyectable tipo medroxiprogesterona en una mujer de 32 años). Coloración PAP x 250.
Las alteraciones celulares por el estudio citológico en casos de infección por VPH mostraron alteraciones morfológicas, como coilocitosis, hipercromacia y binucleación nuclear, disqueratosis y anfofilia citoplasmáticas, señalados ya por los diferentes investigadores (Figura 2).

Santos $\left({ }^{19}\right)$, al igual que otros investigadores, señala que fumar cigarrillos es un cofactor carcinogenético del VPH. Esto no se pudo corroborar en nuestro estudio, pues las mujeres evaluadas no consumían cigarrillos por su baja situación económica.

Hay diferentes opiniones en cuanto a la edad de inicio y término de la detección de cáncer cervical. Bishop $\left({ }^{6}\right)$ y Fajey $\left({ }^{20}\right)$ plantean que es innecesaria la detección en mujeres jóvenes. Y, en programas de recursos limitados, Bishop señala que debería evaluarse a mujeres de 30 a 35 años, entre los 25 y 35 años según Fajey. La mayoría de los investigadores recomiendan que los estudios de detección en cérvix uterino deberían ser realizados entre los 25 y 64 años (Figura 3). Nosotros consideramos importante iniciar la toma de muestras a partir de los 18 años, debido a que la mayoría de las mujeres de las zonas socioeconómicas bajas, especialmente en la selva y sierra, inicia sus relaciones sexuales a temprana edad, muchas antes de los 10 años. Las recomendaciones para la primera prueba de Papanicolaou de los diferentes investigadores señalan realizarla entre los 3 y los 8 años del inicio de las relaciones sexuales.

La nomenclatura usada para la lectura citológica fue la de Bethesda 2001 y, en el caso de biopsias. usamos la clasificación de CIN $\left({ }^{14}\right)$, modificada posteriormente por él mismo a LEI de bajo y alto grado y aprobada por Bethesda en 1990, también como SIL.

El tratamiento de SIL fue hecho en base al diagnóstico histológico y a la extensión de la lesión de acuerdo al examen colposcópico. Las pacientes con L-SIL no requieren tratamiento quirúrgico, debido a que las lesiones 


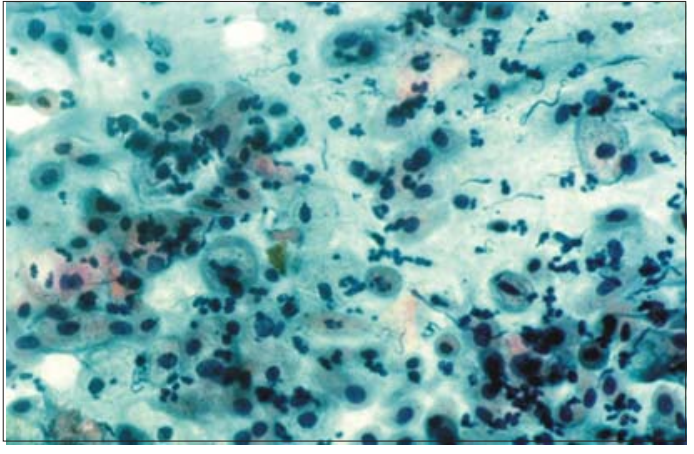

Figura 2. ASC-US: Células pavimentosas parabasales con anisocitosis e hipercromacia nuclear, algunas de ellas con signos de coilocitosis, Fondo con infiltrado inflamatorio agudo, sugiere colpitis atrófica asociada a infección por VPH. Coloración PAP x 400.

mayormente se resuelven espontáneamente con tratamiento médico. Al contrario, las pacientes con H-SIL requieren escisión con Leep o biopsia, para prevenir el posterior desarrollo de carcinoma infiltrante (Figuras 4 y 5). Tuvimos 2 casos de adenocarcinoma, pero su historia natural es diferente a la del carcinoma escamoso, lo cual amerita un estudio especial en un futuro.

Nosotros recomendaríamos la existencia de un ente rector centralizado en un Programa Nacional de Detección de Cáncer Cervical, que reglamente, regule y monitorice el manejo,

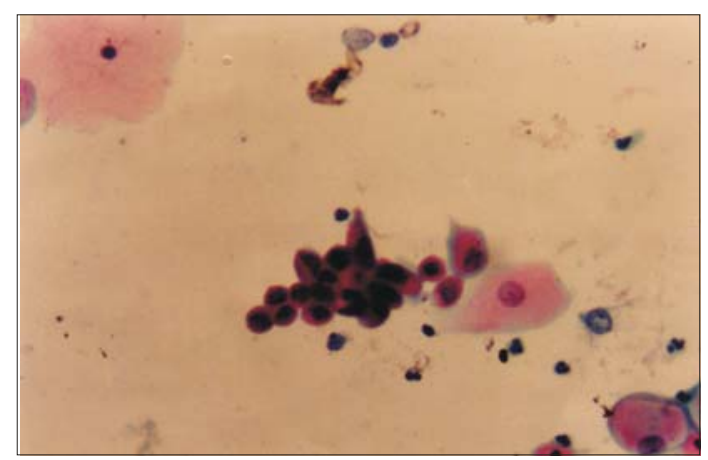

Figura 4. Lesión escamosa intraepitelial de alto grado: Células parabasales inmaduras atípicas con irregularidad de bordes nucleares; disqueratosis citoplasmática. Coloración PAP x 400.

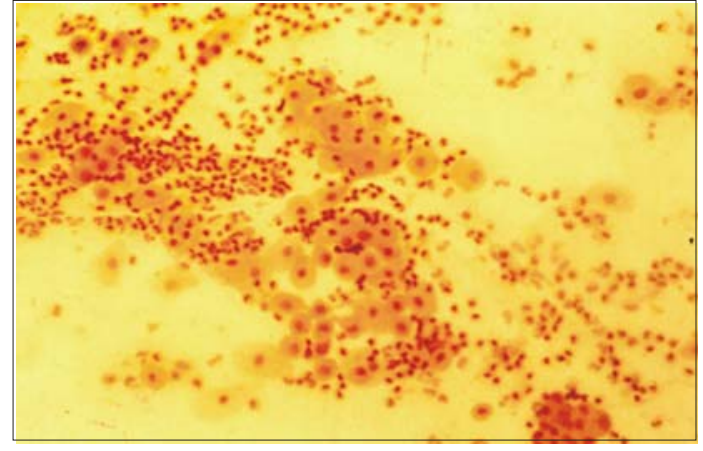

Figura 3. Lesión escamosa intraepitelial de bajo grado: Células pavimentosas intermedias y parabasales con anisocariosis, hipercromasia nuclear y disqueratosis citoplasmática en relación con VPH. Coloración PAP x 400.

control y seguimiento de todos los casos en su fase preclínica, basado no sólo en la citología convencional, sino también el adecuado manejo, seguimiento y tratamiento de la lesión; que otorgue una cobertura de atención nacional, priorizando a la población de mayor riesgo para desarrollar cáncer cervical.

\section{REFERENCIAS BIBLIOGRÁFICAS}

1. Sherris J, Wells E, Tsu V, Bishop A. Cervical cancer in developing countries: A situation analysis. A working paper. Washington DC: World Bank; 1933.

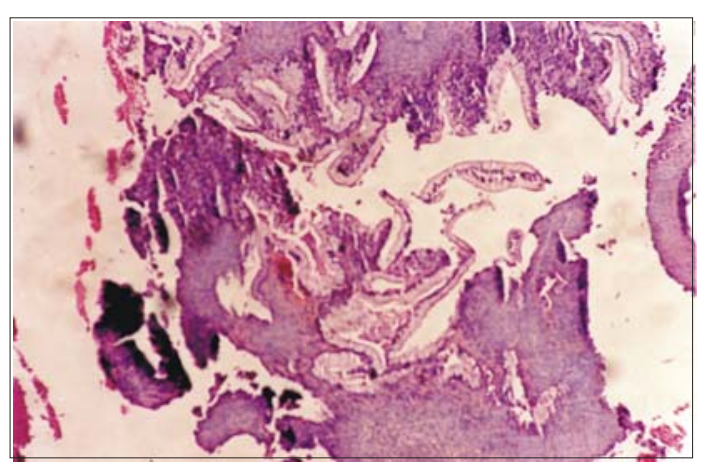

Figura 5. Carcinoma escamoso infiltrante bien diferenciado que compromete glándulas endocervicales. Coloración H.E. x 250. 
2. Parkin DM, Pisani P, Ferlay J. Estimates of the world wide incidence of 18 major cancers in 1985. International Journal of Cancer. 1993;54:594-606.

3. Greenlee RT, Hill Harmon Mb, Murray T, Thun M. Cancer statistics, 2001. Cancer J Clin. 2001;50:7-33.

4. Boffeta P, Parkin DM. Cancer in developing countries. Cancer J Clin. 1994;44:81-90.

5. Centro de Investigación Maes Heller, Instituto de Enfermedades Neoplásicas. Registro de Cáncer en Lima metropolitana, 1990 -1993. Lima: Instituto de Enfermedades Neoplásicas; 1993.

6. Bishop A, Wells E, Sherris J, Tsu V, Crook B. Cervical cancer: evolving prevention strategies for developing countries. Reproductive Health Matters. 1995;(6):60-71.

7. Alba A, Martines MI, Sánchez A, Maestre AM, Tejuca S, Núñez MI, et al. El análisis de la carga viral de los HPVs de alto riesgo tipo 16 y 18 se asocia con el grado de las patologías cervicales. Oncología. 1997;20(5):81-4.

8. Bosch FX, Muñoz N, de Sanjose S. Human papillomavirus and other risk factors for cervical cancer. Biomed Pharmacother. 1997;51(6-7):268-75.

9. Cusik J, Terry G, Monoghan J, López A, Clarkson P. Association between high risk HPV types, HLADRB1 and DQB1, alleles and cervical cancer in British women. Br J Cancer. 2000;82(7):1348-52.

10. Ponten J, Guo Z. Precancer of the human cervix. Cancer Surv. 1998;32:201-29.

11. ANAES. Assessment of human papilloma virus (HPV) testing in primary screening for cervical cancer in France (May 2004). Gynecol Obstet Fertil. 2005;33(5):357-60.

12. Koopman L, Szuhai K, Van Eendenburg JD, Bezrooove VM, Kenter GG, Schuuning E, et al. Recurrent integration papillomaviruses 16,45 and 67 near translocation break points in new cervical cancer cell lines. Cancer Res. 1999;59(21):5615-24.
13. Salomon D, Davey D, Kurman R, Moriarty A, O’Connor D, Prey M, et al. The 2001 Bethesda System: Terminology for reporting results of cervical cytology. JAMA. 2002;287(16):2114-9.

14. Richart RM. A modified terminology for cervical intraepithelial neoplasia. Obstet Gynecol. 1990;75:131-3.

15. Albújar P. El Cáncer en Trujillo 1988-1990: Incidencia y Mortalidad. Lima: Colegio Médico del Perú; 1998. Informe $\mathrm{N}^{\circ} 2$.

16. Koss LG. Cytologic evaluation of the uterine cervix. Factor influencing accuracy. Pathological. 1982;36:401-7.

17. Okagaki T, Fujimura M, Zellerman D. Information discrimination divergence in cytology: III Optimazation of classification of Papnicolaou smears. Acta Cytol. 1991;35:30-5.

18. San Miguel P, Reguera M, Gómez C, Canal C, Antón I, Ortiz-Rey JA, et al. Correlación diagnóstica entre técnicos y patólogos en citologías cérvico-vaginales con lesión escamosa intraepitelial. Vigo: Centro Médico Povisa; 2001.

19. Santos C, Munoz N, Klug S, Almonte M, Guerrero I, Alvarez M, et al. HPV types and cofactors causing cervical cancer in Peru. Br J Cancer. 2001;85(7):966-71.

20. Fahey MT, Irwig L, Macaskill P. Meta-analysis of Pap test accuracy. Am J Epidemiol. 1995;141(7):680-9.

Manuscrito recibido el 15 de marzo de 2005 y aceptado para publicación el 02 mayo de 2005.

Correspondencia: Dra. Victoria Eugenia Valer Tito

Av. Uruguay 126 Dpto. 201.

Lima 1, Perú.

Correo-e:mirandavaler@yahoo.com 